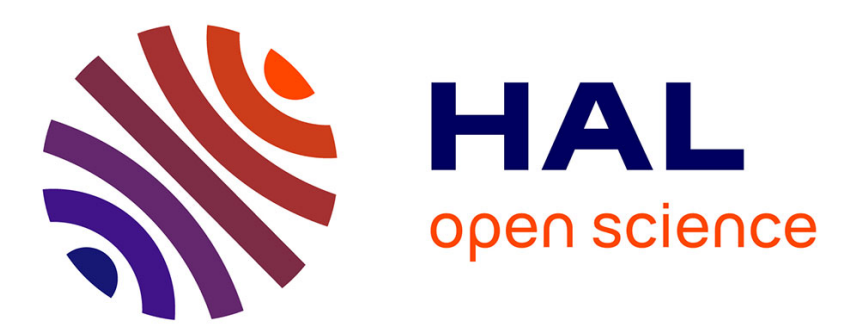

\title{
Apport des méthodes d'enrichissement sélectives et des spectrométries à l'identification des constituants minéraux d'un échantillon de sol
}

\author{
Ary Bruand, René Prost
}

\section{- To cite this version:}

Ary Bruand, René Prost. Apport des méthodes d'enrichissement sélectives et des spectrométries à l'identification des constituants minéraux d'un échantillon de sol. Agronomie, 1986, 6 (8), pp.717-726. hal-00884929

\section{HAL Id: hal-00884929 \\ https://hal.science/hal-00884929}

Submitted on 1 Jan 1986

HAL is a multi-disciplinary open access archive for the deposit and dissemination of scientific research documents, whether they are published or not. The documents may come from teaching and research institutions in France or abroad, or from public or private research centers.
L'archive ouverte pluridisciplinaire HAL, est destinée au dépôt et à la diffusion de documents scientifiques de niveau recherche, publiés ou non, émanant des établissements d'enseignement et de recherche français ou étrangers, des laboratoires publics ou privés. 


\title{
Apport des méthodes d'enrichissement sélectives et des spectrométries à l'identification des cons- tituants minéraux d'un échantillon de sol
}

\author{
Ary BRUAND \& René PROST \\ I.N.R.A., Station de Science du Sol, route de St-Cyr, F 78000 Versailles
}

RÉSUMÉ

\begin{abstract}
Les matériaux du sol sont constitués de particules de taille et de cristallinité variables. L'exceptionnelle réactivité des particules les plus fines ou les plus mal cristallisées explique l'intérêt qu'on attache à leur identification. On montre comment, à partir de l'exemple choisi, l'association des spectrométries d'absorption et de résonance (infrarouge, Mössbauer, ...) aux techniques plus classiques comme la diffraction des RX permet de révéler l'existence de certains constituants présents dans le mélange et conduit à en préciser la nature. On montre, par ailleurs, que la présence en très petite quantité de certains composés oblige à procéder à des séparations granulométriques et à des dissolutions sélectives d'une ou plusieurs phases minérales. Ces traitements ont pour effet d'enrichir l'échantillon analysé en un ou plusieurs constituants. La présente étude montre qu'une caractérisation plus complète des matériaux du sol implique la mise en œuvre d'un éventail élargi de techniques et de traitements spécifiques des échantillons ; ces derniers étant choisis en fonction de la nature du matériau à caractériser.
\end{abstract}

Mots clés additionnels : Séparation granulométrique, dissolution sélective, infrarouge, Mössbauer, réflexion diffuse, diffraction $R X$, argile, oxyhydroxyde.

Soil materials are made of particles of varied size and crystallinity. The high reactivity of the smallest or poorly crystallized particles explains the interest which attaches to their identification. The present work has shown how, by using both spectrometric methods (infrared, Mössbauer, ...) and X-ray diffraction, it is possible to reveal the existence of components in the analysed sample and to identify them precisely. Moreover, because of the presence of small quantities of certain components in the sample, size fractionations and selective dissolutions have been carried out. These treatments made it possible to enrich the sample with respect to one or several constituents. Thus, in order to obtain complete characterization of a soil material, a variety of methods and specific treatments of the sample have to be carried out and adapted to the nature of the soil material.

Additional key words : Size fractionation, selective dissolution, infrared, Mössbauer, diffuse reflectance, $X$ ray diffraction, clay, iron hydroxide.

\section{INTRODUCTION}

La caractérisation de la phase minérale du sol est nécessaire pour comprendre les propriétés de surface et, par voie de conséquence, les interactions qui existent entre les différents constituants qui le composent. On utilise pour cela la diffraction des RX, les analyses thermiques et les microscopies optiques ou électroniques; l'analyse chimique totale complétée par la détermination de certaines propriétés physicochimiques (surface spécifique, capacité d'échange, ...) donne l'ensemble des déterminations sur lesquelles repose généralement l'analyse (CAILLĖRE et al., 1982).
Les techniques utilisées présentent des limites. Elles résultent, pour certaines, du caractère global des déterminations (analyse chimique, capacité d'échange, ...) qui sont faites sur des mélanges. Il faut pour affiner les résultats et parvenir à une identification des constituants procéder à la séparation ou, au moins, à l'enrichissement de certains constituants. Pour d'autres techniques, la limite est une conséquence du principe physique sur lequel s'appuie la détermination. Ainsi, dans le cas de la diffraction des RX, les domaines atomiques rencontrés par le rayonnement $\mathrm{X}$ doivent être suffisamment grands pour que les ondes diffusées par chaque atome puissent former un phéno- 
mène d'interférence détectable. Par conséquent, seules les particules présentant un ordre atomique à grande distance donnent un signal ; ce qui explique que la diffraction des RX ne soit pas adaptée à la détection des particules nanométriques ou mal cristallisées. Cette limite est d'autant plus gênante que les échantillons de sol contiennent des particules nanométriques, comme certains composés du fer ou de l'aluminium, mais aussi des minéraux formés par l'empilement plus ou moins ordonné de feuillets à 0,7 ou $1 \mathrm{~nm}$ d'épaisseur. L'ordre atomique au sein de ces composés, qui correspondent à différents stades des processus d'altération ou de formation, est éminemment variable d'un constituant à l'autre. On montre par exemple qu'un ordre à très courte distance entre un atome et ses premiers voisins, qui préfigure le cristal, existe dès les premiers stades de sa formation (DECARREAU, 1983). De la même manière on montre que l'ordre cristallin est perturbé aux limites des cristaux (RAUTUREAU \& TCHOUBAR, 1974).

En conséquence, pour caractériser ces petites particules ou ces composés, où seul un ordre cristallin à courte distance existe, il faut mettre en œuvre des techniques adaptées comme, par exemple, les spectrométries d'absorption (Extended X-ray Absorption Fine Structure ou EXAFS, infrarouge, ...) ou de résonance (RMN, Mössbauer, ...). En effet, l'extrême sensibilité des techniques spectrométriques aux relations entre les atomes conduit généralement, pour chacun des constituants présents dans l'échantillon, à un signal caractéristique qui peut être utilisé pour l'identification des constituants d'un mélange.

On se propose dans la suite de montrer, d'une part, comment l'utilisation de techniques d'extraction appropriées pour séparer ou enrichir le système en un ou plusieurs constituants augmente les performances des techniques utilisées habituellement et, d'autre part, comment l'utilisation de techniques spectrométriques comme l'absorption infrarouge ou le Mössbauer permet, associées aux précédentes, de préciser la nature des différents constituants d'un échantillon de sol.

\section{MATÉRIEL}

L'échantillon étudié (tabl. 1) provient de l'horizon B d'un profil de sol de "terre d'Aubues" (BRUAND, 1985). Ces sols, développés sur les plateaux jurassiques calcaires à la limite sud-est du bassin parisien, ont été étudiés par BAIZE $(1971,1972 a$ et $b$, 1976). Les agrégats conservés humides (teneur en eau pondérale de $25 \mathrm{p}$. 100) sont dispersés dans l'eau après avoir été forcés à travers un tamis de $50 \mu \mathrm{m}$. La fraction $<2 \mu \mathrm{m}$ utilisée dans la suite de ce travail
(Ech. $<2 \mu \mathrm{m}$ ) est extraite par sédimentation de cette suspension. Les extractions sont faites sans prétraitement dispersant pour éviter le risque de polluer ou d'éliminer un des constituants du mélange.

\section{MÉTHODES}

En raison des proportions très différentes de chacun des constituants dans le mélange et des limites des méthodes physiques d'analyse, on a cherché à enrichir l'échantillon en l'une ou l'autre des phases en présence en procédant à une séparation granulométrique ou à la dissolution d'un de ces constituants.

\section{A. Préparation de l'échantillon}

La séparation des particules en fonction de leur taille est faite par centrifugation d'une suspension à $1 \mathrm{~g} .1^{-1}$ à l'aide d'un bol à flux continu. Les fractions $2-0,2 \mu \mathrm{m}$ (Ech. 2-0,2 $\mu \mathrm{m}$ ) et $<0,2 \mu \mathrm{m}$ (Ech. $<0,2 \mu \mathrm{m})$ sont extraites. Elles représentent respectivement 56 et 44 p. 100 de l'echantillon. On montre dans la suite que cette coupure granulométrique permet l'élimination de la plus grande partie des grains de quartz tout en n'altérant pas les autres constituants du mélange.

La dissolution sélective de la kaolinite est effectuée à l'aide d'une méthode mise au point par KAMPF \& SCHWERTMANN (1982). Ce traitement, qui consiste à faire bouillir pendant une heure l'échantillon dispersé dans la soude $5 \mathrm{M}$, vise à concentrer les composés du fer.

Enfin, la dissolution des oxyhydroxydes de fer est faite à l'aide d'un mélange d'acide oxalique et d'oxalate d'ammonium à $\mathrm{pH}=3$; le mélange étant soumis à un rayonnement ultraviolet (DE ENDREDY, 1963 ; SCHWERTMANN, 1964 ; SCHWARZMANN \& SPARR, 1969). Ce traitement, effectué sur la fraction $<0,2 \mu \mathrm{m}$ après avoir dissout la kaolinite, enrichit l'échantillon en minéraux argileux.

\section{B. Méthodes d'étude}

Les échantillons obtenus sont étudiés par diffraction des RX sous forme de poudre en utilisant la raie $\mathrm{K}_{x}$ du cobalt $(\lambda=1,789 \AA)$ ou sous forme de dépôt orienté sur des lames de verre en utilisant la raie $\mathrm{K}_{\alpha} \mathrm{du}$ cuivre ( $\lambda=1,541 \AA$ ). L'utilisation d'une cellule (fig. 1) a permis soit de contrôler l'humidité relative avec laquelle l'échantillon est en équilibre, soit de sou" mettre cet échantillon au vide d'une pompe à palette et de le chauffer.

TABLEAU 1

Analyses chimiques de Ech. $<2 \mu \mathrm{m}$.

Chemical analysis of the $<2 \mu \mathrm{m}$ sample.

\begin{tabular}{|c|c|c|c|c|c|c|c|c|c|c|c|c|c|c|}
\hline \multirow{2}{*}{$\begin{array}{c}\text { M.O. } \\
\%\end{array}$} & \multirow{2}{*}{$\begin{array}{c}\text { C.E.C. } \\
\text { meq } / 100 \mathrm{~g}\end{array}$} & \multicolumn{4}{|c|}{ Cations échangeables meq $/ 100 \mathrm{~g}$} & \multirow{2}{*}{$\begin{array}{c}\text { Fer } \\
\text { Deb } \\
\% 0 \\
\end{array}$} & \multicolumn{7}{|c|}{ Eléments totaux (HF) \% pondéral de l'échantillon calciné } & \multirow{2}{*}{$\begin{array}{l}\text { Fer Deb } \\
\text { Fer tota }\end{array}$} \\
\hline & & $\mathrm{Ca}$ & $\mathrm{Mg}$ & $\mathrm{K}$ & $\mathrm{Na}$ & & $\mathrm{SiO}_{2}$ & $\mathrm{Al}_{2} \mathrm{O}_{3}$ & $\mathrm{Fe}_{2} \mathrm{O}_{3}$ & $\mathrm{MgO}$ & $\mathrm{CaO}$ & $\mathrm{Na}_{2} \mathrm{O}$ & $\mathrm{K}_{2} \mathrm{O}$ & \\
\hline 1,0 & 34,1 & 30,1 & 1,7 & 0,9 & 0,4 & 5,27 & 53,34 & 29,11 & 12,54 & 1,44 & 1,00 & 0,09 & 2,48 & 0,68 \\
\hline
\end{tabular}



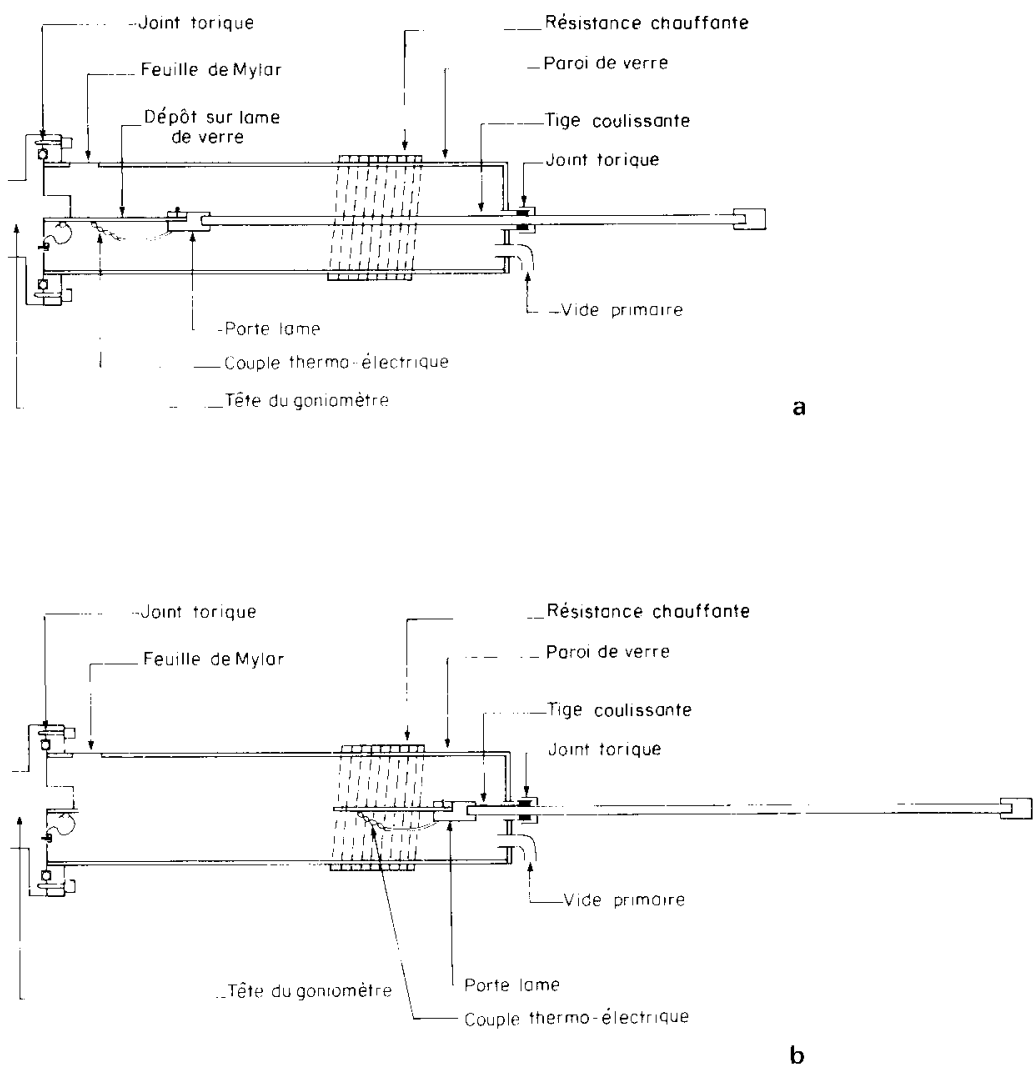

Figure 1

Cellule utilisée pour la diffraction des RX. L'échantillon est: (a) placé pour l'enregistrement, (b) amené au niveau du four en faisant coulisser la tige porte-échantillon.

Les études par spectrométrie sont réalisées en réflexion diffuse, à l'aide du spectromètre Beckman 5270 U.V., visible et proche infrarouge équipé de sa sphère d'intégration. L'échantillon est placé dans une cellule étanche. Son épaisseur est dans tous les cas supérieure à $2 \mathrm{~mm}$ pour qu'il puisse être considéré, du point de vue de la réflexion diffuse, comme un milieu semi-infini. Les spectres représentent $\log (\mathrm{Ro} / \mathrm{R})$ en fonction de la longueur d'onde exprimée en $\mathrm{nm}$. L'examen par spectrométrie infrarouge est réalisé en employant la technique des pastilles $\mathrm{KBr}$. Le spectromètre utilisé est un Perkin Elmer 580 équipé d'un dispositif permettant l'élimination de l'eau et du gaz carbonique de l'air. Quant à la spectrométrie Mössbauer qui est spécifique du fer, les spectres ont été réalisés à $298^{\circ} \mathrm{K}$ et $80^{\circ} \mathrm{K}$ au Laboratoire de Chimie de la Matière Condensée (E.N.S.C.P.).

\section{RÉSULTATS ET DISCUSSION}

Les diffractogrammes des Ech. $<2 \mu \mathrm{m}$, Ech. $2-0,2 \mu \mathrm{m}$ et Ech. $<0,2 \mu \mathrm{m}$ sous forme de poudre (fig. 2) montrent la présence des raies caractéristiques du quartz $(4,26 ; 3,34$ et $2,46 \AA)$, de la kaolinite $(7,19 ; 3,57$ et $2,34 \AA)$ et de minéraux argileux $2: 1$ (raies larges pour lesquelles les maxima apparents sont à $13,9 \AA, 11,8 \AA$ et $4,96 \AA$ et des raies plus fines à 4,49 et $2,56 \AA$ ) (BRINDLEY \& BROWN, 1980). La comparaison des spectres a et $\mathrm{c}$ de la figure 2 montre que
Cell used for X-ray diffraction. The sample is deposited on a glass slide which is: (a) placed at the head of the goniometer to record the spectrum or (b) put at oven level, sliding the specimen rod.

la coupure granulométrique effectuée à $0,2 \mu \mathrm{m}$ permet l'élimination de la plus grande partie des grains de quartz alors que les autres constituants restent présents dans le mélange. La figure 2-b indique que Ech. $2-0,2 \mu \mathrm{m}$ contient, en plus des grains de quartz, une proportion tout à fait détectable des autres constituants du mélange. On peut dire que le fractionnement granulométrique conduit à un enrichissement de Ech. 2-0,2 $\mu \mathrm{m}$ en quartz et, par voie de conséquence, à un appauvrissement en ce minéral de Ech. $<0,2 \mu \mathrm{m}$.

Par ailleurs, bien que 68 p. 100 du fer total soient extractibles par la méthode DEB (1950) (tabl. 1) et qu'ils doivent donc être attribués à du fer n'appartenant pas aux minéraux argileux, la diffraction des RX ne permet pas de déceler la présence de composés du fer, même dans le cas où l'échantillon est débarrassé du quartz (fig. 2-c).

Une caractérisation plus précise des constituants nécessite d'autres prétraitements et d'autres techniques d'analyse.

\section{A. La kaolinite}

L'examen des spectres présentés figure 3-a et 4-a révèle l'absence du doublet à $780-798 \mathrm{~cm}^{-1}$ caractéristique du quartz sur le spectre de Ech. $<0,2 \mu \mathrm{m}$. Ceci montre que le fractionnement granulométrique effectué a permis l'élimination de la plus grande partie du quartz. 


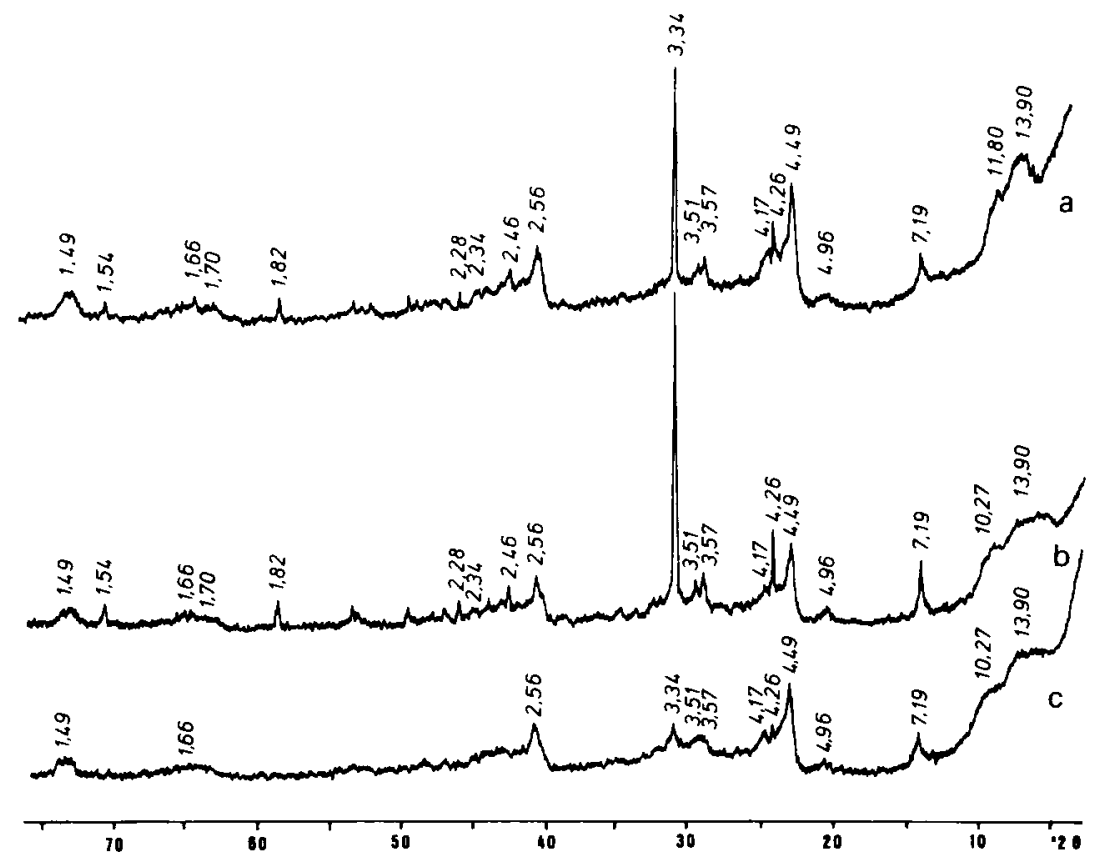

Figure 2

Diffractogrammes $R X\left(K_{\alpha}\right.$ Co $; H R=50$ p. 100 ; poudre non orientée) : (a) Ech. < $2 \mu \mathrm{m}$, (b) Ech. $2-0,2 \mu \mathrm{m}$ et (c) Ech. < 0,2 $\mu \mathrm{m}$. Ces échantillons sont saturés par $\mathrm{Ca}^{++}$. Les valeurs portées à la verticale des pics sont exprimées en angström.

$X$-ray powder diffractograms $(\mathrm{CoK} ; R H=50 \% ;$ satured with $\left.\mathrm{Ca}^{++}\right):(a)<2 \mu \mathrm{m}$ sample, (b) $0.2-2 \mu \mathrm{m}$ sample and $(c)<0.2 \mu \mathrm{m}$ sample. The numbers above each line are in $\mathrm{A}$.
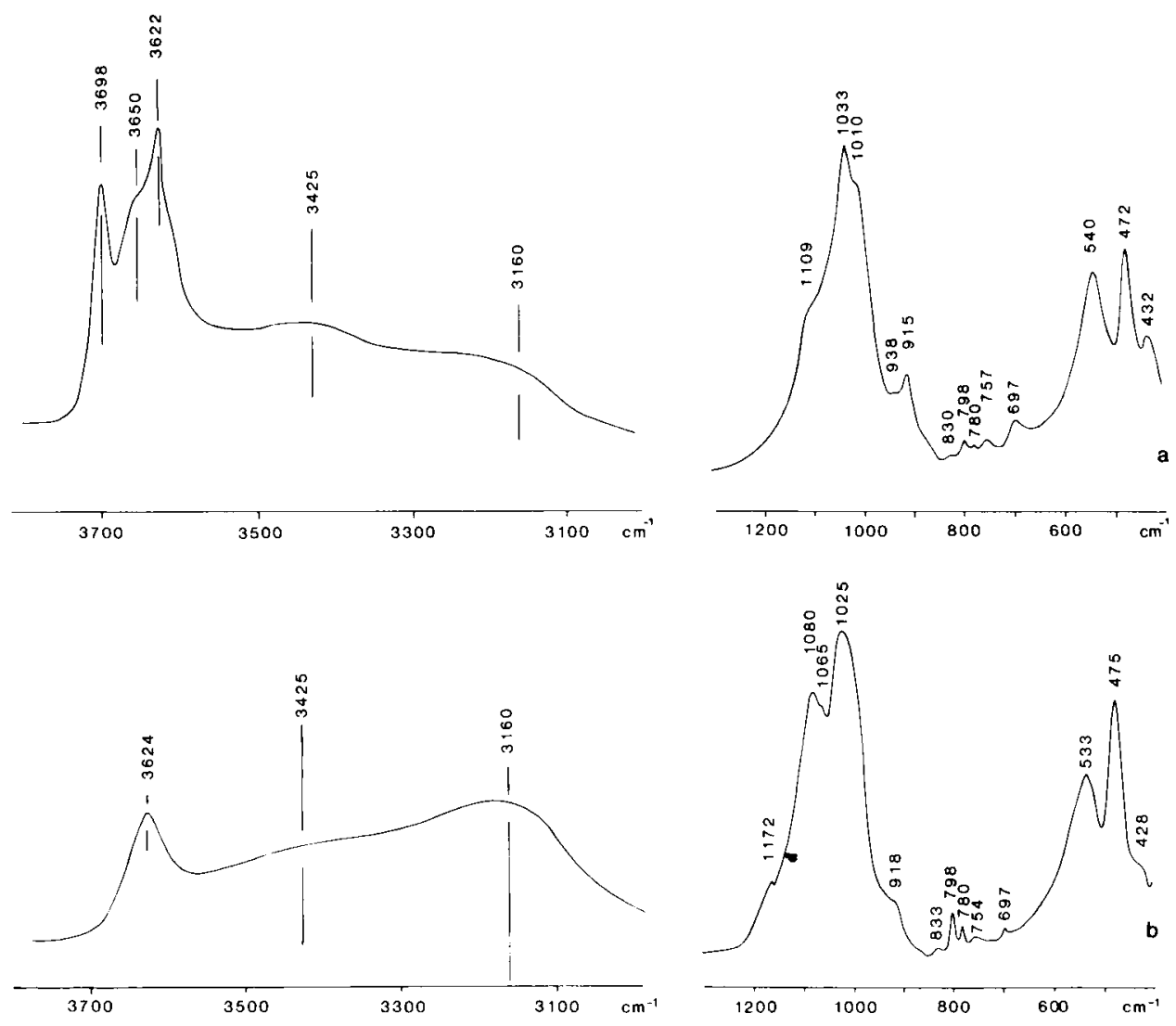

Figure 3

Spectres infrarouge de Ech. $<2 \mu \mathrm{m}$ saturé par $\mathrm{Ca}^{++}:$(a) chauffé à $105^{\circ} \mathrm{C}$, (b) traité par $\mathrm{NaOH} 5 \mathrm{M}$ et chauffé à $105^{\circ} \mathrm{C}$.

Infrared spectra of the $<2 \mu \mathrm{m}$ sample saturated with $\mathrm{Ca}^{++}$: (a) heated to $105^{\circ} \mathrm{C}$, (b) after treatment with $5 \mathrm{M} \mathrm{NaOH}$ and heated to $105^{\circ} \mathrm{C}$.

\section{Figure 4}

Spectres infrarouge: (a) Ech. $<0,2 \mu \mathrm{m}$ saturé par $\mathrm{Ca}^{++}$et chauffé à $105^{\circ} \mathrm{C}$, (b) Ech. $<0,2 \mu \mathrm{m}$ traité par $\mathrm{NaOH} 5 \mathrm{M}$ et chauffé à $105^{\circ} \mathrm{C}$, (c) Ech. $<0,2 \mu \mathrm{m}$ traité par $\mathrm{NaOH} 5 \mathrm{M}$ puis par le traitement de De Hendredy, saturé avec $\mathrm{NH}_{4}^{+}$et chauffé à $105^{\circ} \mathrm{C}$. (d) la fraction < $2 \mu \mathrm{m}$ de l'illite du Puy.
Infrared spectra : (a) $<0.2 \mu \mathrm{m}$ sample saturated with $\mathrm{Ca}^{++}$and heated to $105^{\circ} \mathrm{C}$, (b) $<0.2 \mu \mathrm{m}$ sample treated with $5 \mathrm{M} \mathrm{NaOH}$ and heated to $105{ }^{\circ} \mathrm{C}$, (c) $<0.2 \mu \mathrm{m}$ sample treated with $5 \mathrm{M} \mathrm{NaOH}$ then with De Endredy treatment, satured with $\mathrm{NH}_{4}^{+}$and heated to $105{ }^{\circ} \mathrm{C},(d)<2 \mu \mathrm{m}$ fraction of "Illite du Puy". 

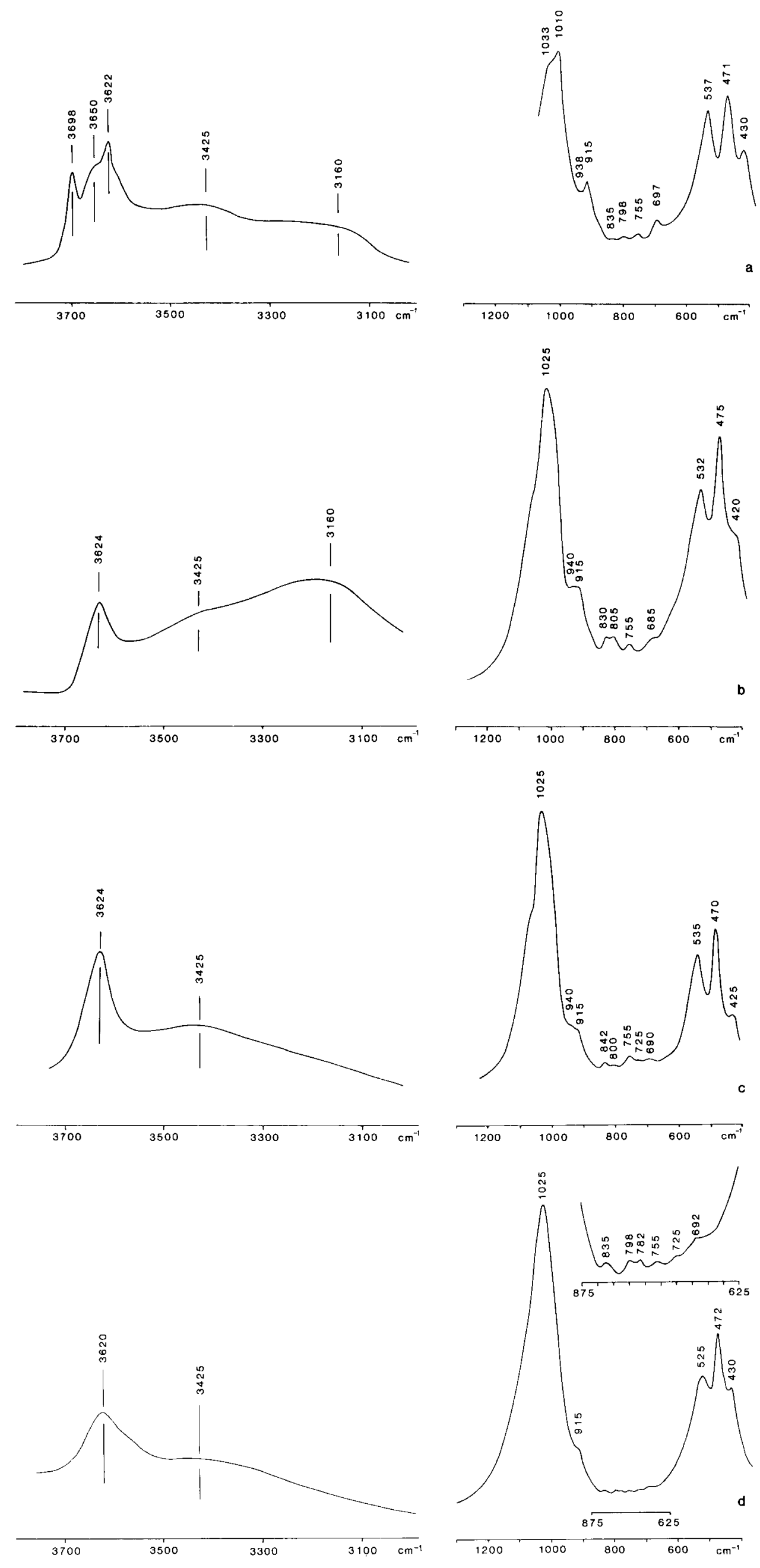
Le spectre infrarouge des groupes $\mathrm{OH}$ de Ech. $<2 \mu \mathrm{m}$ présente 2 bandes à 3698 et $3622 \mathrm{~cm}^{-1}$ dans le domaine des vibrations de valence et 2 autres à $915 \mathrm{~cm}^{-1}$ et $938 \mathrm{~cm}^{-1}$ dans le domaine des vibrations de déformation angulaire (fig. 3-a) qui correspondent aux vibrations des hydroxyles de la kaolinite (FARMER, 1974 ; ROUXHET et al., 1977). Le spectre infrarouge des hydroxyles de structure de Ech. $<0,2 \mu \mathrm{m}$ présente les mêmes caractéristiques (fig. 4-a). Ceci indique que ces 2 échantillons contiennent le même type d'argile.

\section{B. Les minéraux argileux 2:1}

Pour identifier les minéraux argileux 2:1, on cherche à préciser leur caractère di- ou trioctaédrique, la valeur de leur charge globale et sa localisation (PEDRO, 1967 ; CAILlÈre et al., 1982).

L'absence de raie à $14 \AA$ sur le diffractogramme de Ech. $<2 \mu \mathrm{m}$ saturé par du $\mathrm{Mg}^{++}$et chauffé à $220{ }^{\circ} \mathrm{C}$ sous vide (fig. 5-d) permet de conclure à

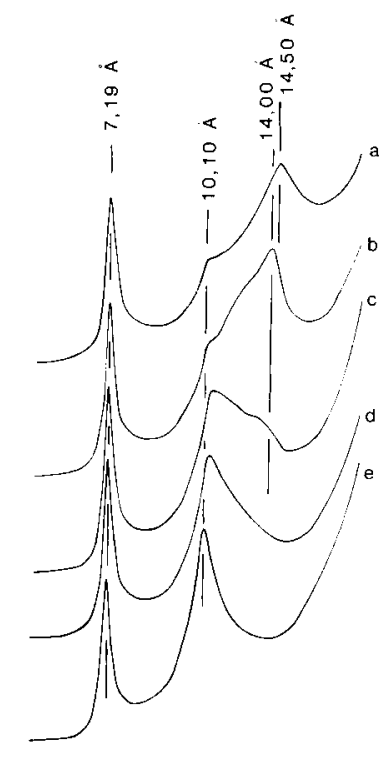

Figure 5

Diffractogrammes $R X\left(K_{\alpha} C u\right.$; dépôt orienté) de Ech. $<2$ um saturé par $\mathrm{Mg}^{++}$: (a) placé dans une atmosphère où $H R=55 \mathrm{p} .100$, (b) sous un vide de $10^{-2}$ Torr, (c) chauffé sous vide à $105^{\circ} \mathrm{C}$, (d) chauffé sous vide à $220^{\circ} \mathrm{C}$, (e) chauffé I $h$ dans un four à $400{ }^{\circ} \mathrm{C}$.

$X$-ray diffractograms $\left(\mathrm{Cu} K_{c x}\right.$; oriented deposit; saturated with $\mathrm{Mg}^{++}$) of the $<2 \mu \mathrm{m}$ sample : (a) $\mathrm{RH}=55 \%$, (b) under $a$ vacuum of $10^{-2}$ Torr, (c) under vacuum and heated to $105^{\circ} \mathrm{C}$, (d) under vacuum and heated to $220^{\circ} \mathrm{C}$, (e) oven heated to $400^{\circ} \mathrm{C}$.

l'absence de minéraux intergrades. Lorsque cet échantillon est saturé par $\mathrm{K}^{+}$, une seule mise sous vide suffit pour faire disparaître la raie à $14 \AA$ (fig. 6-b), ce qui confirme l'absence de minéraux intergrades.

\section{Etude du caractère di- ou trioctaédrique}

Le caractère di- ou trioctaédrique est aisément vérifié par spectrométrie IR en étudiant la fréquence des bandes de vibration de valence des hydroxyles de structure des minéraux argileux (tabl. 2). Le spectre obtenu avec Ech. $<2 \mu \mathrm{m}$ ne possède pas de bande de

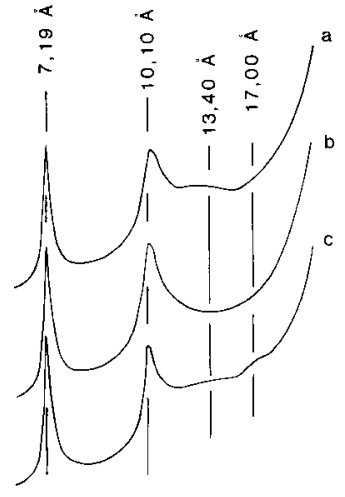

Figure 6

Diffractogrammes $R X\left(K_{\alpha} C u\right.$; dépôt orienté) de Ech. $<2 \mu \mathrm{m}$ saturé par $K^{+}:$(a) placé dans une atmosphère où $H R=50 \mathrm{p} .100$, (b) sous un vide de $10^{-2}$ Torr, (c) chauffé à $105^{\circ} \mathrm{C}$ puis saturé avec de l'éthylène glycol.

$X$-ray diffractograms (Cu $K_{\alpha}$; oriented deposit) of the $<2 \mu \mathrm{m}$ sample saturated with $K^{+}:$(a) $R H=50 \%$, (b) under vacuum of $10^{-2}$ Torr, (c) heated to $105^{\circ} \mathrm{C}$ then saturated with ethylene glycol.

vibration de déformation angulaire décelable à $650 \mathrm{~cm}^{-1}$ caractéristique des minéraux trioctaédriques mais, par contre, 2 bandes à 915 et à $938 \mathrm{~cm}^{-1}$ (fig. 3-a) caractéristiques des minéraux dioctaédriques, en particulier de la kaolinite. Afin de préciser la nature du minéral associé à la kaolinite, on élimine celle-ci par le traitement $\mathrm{NaOH} 5 \mathrm{M}$. La présence d'une bande de vibration de valence à $3624 \mathrm{~cm}^{-1}$ et d'une bande de vibration de déformation à $918 \mathrm{~cm}^{-1}$ (fig. 3-b), permet d'affirmer que les minéraux argileux $2: 1$ présents dans Ech. $<2 \mu \mathrm{m}$ après le traitement $\mathrm{NaOH} 5 \mathrm{M}$ sont dioctaédriques.

Le caractère dioctaédrique est confirmé sur le spectre de réflexion diffuse obtenu après traitement de Ech. $<2 \mu \mathrm{m}$ par NaOH 5M (fig. 7-b). Ce spectre présente, dans le domaine $2100-2500 \mathrm{~nm}$, une seule bande d'absorption à $2200 \mathrm{~nm}$ qui est due à la combinaison des vibrations de déformation angulaire $\left(930 \mathrm{~cm}^{-1}\right)$ et de valence $\left(3620 \mathrm{~cm}^{-1}\right)$ des groupements $\mathrm{OH}$ des minéraux argileux 2:1 (HUNT et al., 1973). Un minéral trioctaédrique donnerait des bandes à 2310 et $1360 \mathrm{~nm}$ qu'on ne détecte pas (fig. 7-a et 7b, tabl. 2).

\section{Etude des relations solvant-cation-feuillet de l'argile}

Cette étude est réalisée à partir de Ech. $<2 \mu \mathrm{m}$ sous forme de dépôt orienté dans des conditions contrôlées grâce à la cellule utilisée pour la diffraction des RX (fig. 1).

L'examen des spectres de diffraction des RX de Ech. $<2 \mu \mathrm{m}$, saturé par du $\mathrm{Mg}^{++}$et pour différents états d'hydratation (fig. 5), montre que certains espaces interfoliaires demeurent fermés $\left(d_{001}=10,10 \AA\right)$ alors que d'autres gonflent $\left(\mathrm{d}_{001}>10,10 \AA\right)$. Ce type de comportement permet de séparer un premier ensemble de feuillets à espaces interfoliaires anhydres quelles que soient la nature du cation échangeable et celle du solvant. La charge globale de ces feuillets est supérieure à 0,6 par maille et ils sont probablement saturés par des cations $\mathrm{K}^{+}$(ROBERT, 1975). Pour les autres feuillets dont le déficit de charge est saturé par 
TABLEAU 2

Fréquence des bandes d'absorption infrarouge et proche infrarouge des hydroxyles de structure des argiles. Infrared and near infrared absorption band frequencies of clay structural OH groups.

\begin{tabular}{|c|c|c|c|c|c|c|c|c|c|}
\hline \multirow{3}{*}{\multicolumn{2}{|c|}{$\begin{array}{l}\text { Longueur d'onde } \\
\text { déterminée } \\
\text { à partir } \\
\text { du spectre }\end{array}$}} & \multicolumn{4}{|c|}{ Vibrations fondamentales } & \multicolumn{4}{|c|}{ Vibrations harmoniques } \\
\hline & & \multicolumn{2}{|c|}{$\begin{array}{c}\text { Vibration de } \\
\text { déformation angulaire } \\
\delta\end{array}$} & \multicolumn{2}{|c|}{$\begin{array}{l}\text { Vibration de } \\
\text { valence }\end{array}$} & \multirow{2}{*}{\multicolumn{2}{|c|}{$\delta+\gamma$}} & \multirow[b]{2}{*}{$\mathrm{cm}^{-1}$} & \multirow[b]{2}{*}{$\mathrm{nm}$} \\
\hline & & $\mathrm{cm}^{-1}$ & $\mathrm{~nm}$ & $\mathrm{~cm}^{-1}$ & $\mathrm{~nm}$ & & & & \\
\hline 1920 & 1450 & 1650 & 6060 & 3450 & 2900 & 5100 & 1960 & 6900 & 1450 \\
\hline \multirow[t]{2}{*}{2200} & 1408 & 930 & 10753 & 3620 & 2762 & 4550 & 2200 & 7240 & 1380 \\
\hline & & 650 & & 3680 & & 4330 & 2310 & 7360 & 1360 \\
\hline
\end{tabular}

des cations $\mathrm{Mg}^{++}$, le profil de la raie à $14,50 \AA$ pour $\mathrm{HR}=55 \mathrm{p} .100$, ainsi que la fermeture progressive et non homogène des différents espaces interfoliaires intervenant lors du chauffage, montrent que le minéral ne forme pas avec l'eau un complexe régulier (fig. 5).

La nature des complexes formés avec l'eau ou les solvants organiques dépend de la valeur et de la localisation des déficits de charges positives dans le feuillet et de la nature des cations compensateurs (BRINDLEY \& BROWN, 1980). Ainsi, quand on procède à un échange des cations $\mathrm{Mg}^{++}$par $\mathrm{K}^{+}$et de l'eau par l'éthylène glycol, le diffractogramme obtenu (fig. 6-c)

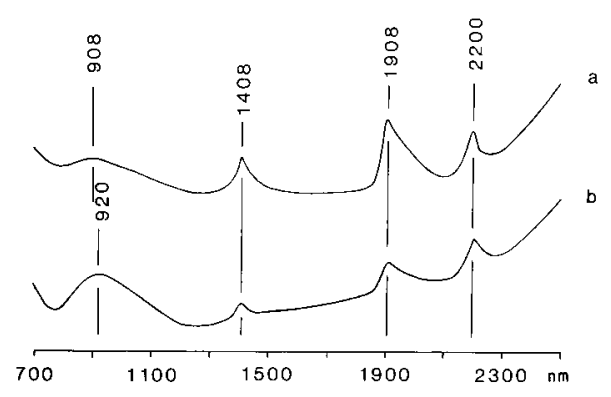

Figure 7

Spectres de réflexion diffuse dans le proche infrarouge de Ech. $<2 \mu \mathrm{m}$ (a) avant et (b) après traitement avec NaOH $5 \mathrm{M}$.

Reflectance spectra in the near infrared range of the $<2 \mu \mathrm{m}$ sample (a) before and (b) after the treatment with $5 \mathrm{M} \mathrm{NaOH}$.

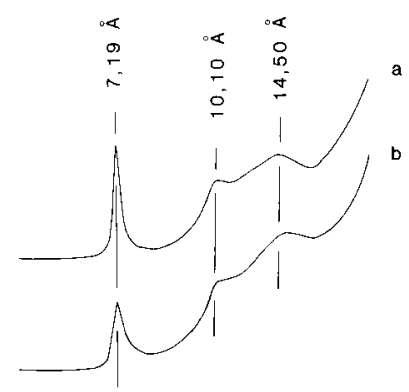

Figure 8

Diffractogrammes $R X\left(K_{\alpha} C u ; H R=55 p .100\right.$; dépôt orienté :

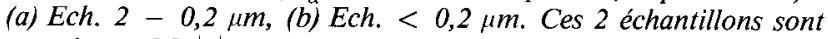
saturés par $\mathrm{Mg}^{++}$.

$X$-ray diffractograms $\left(\mathrm{Cu} K_{\alpha} ; R H=55 \%\right.$; oriented deposit) : (a) $2-0.2 \mu \mathrm{m}$ sample and (b) $<0.2 \mu \mathrm{m}$ sample. These two samples are satured with $\mathrm{Mg}^{++}$.

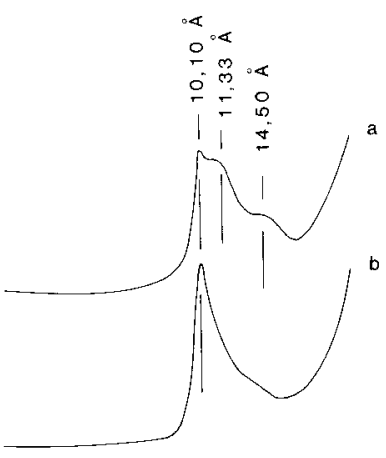

Figure 9

Diffractogrammes $R X\left(K_{\alpha} C u\right.$; dépôt orienté) de Ech. $<2 \mu \mathrm{m}$ traité avec $\mathrm{NaOH} 5 \mathrm{M}$ et saturé par $\mathrm{Mg}^{++}:$(a) placé dans une atmosphère où $H R=55 \mathrm{p} .100$, (b) chauffé à $105^{\circ} \mathrm{C}$ sous vide.

$X$-ray diffractograms (Cu $K_{\alpha}$; oriented deposit) of the $<2 \mu \mathrm{m}$ sample treated with $5 \mathrm{M} \mathrm{NaOH}$ then saturated with $\mathrm{Mg}^{++}$: (a) $R H=55 \%$, (b) under vacuum and heated to $105{ }^{\circ} \mathrm{C}$.

montre que, les feuillets, excepté un très petit nombre (raies à 17 et $13,40 \AA$ ), ne peuvent gonfler après avoir été chauffés à $105^{\circ} \mathrm{C}$. La valeur de la charge du feuillet est alors estimée supérieure à 0,6 par maille (ROBERT, 1975). Néanmoins, pour ces feuillets et ceux à espace interfoliaire non gonflant, on ne peut conclure quant à la répartition de la charge en couche tétraédrique et/ou octaédrique.

\section{Discussion}

La caractérisation des minéraux présents dans les échantillons a été faite à l'aide de la diffraction des RX et des spectrométries infrarouge. Cette analyse a été conduite en éliminant successivement le quartz par séparation granulométrique et la kaolinite par dissolution sélective. On peut naturellement s'interroger sur la représentativité des minéraux argileux 2:1 obtenus à l'issue de tels traitements. En ce qui concerne le fractionnement granulométrique, l'allure des diffractogrammes RX (fig. 5-a et 8-b) et des spectres infrarouge (fig. 3-a et 4-a) obtenus ne permet pas de révéler de différence entre les minéraux argileux 2:1 de Ech. $<2 \mu \mathrm{m}$ et ceux de Ech. $<0,2 \mu \mathrm{m}$. Quant aux minéraux argileux 2:1 obtenus à la suite de l'élimination de la kaolinite par le traitement $\mathrm{NaOH} 5 \mathrm{M}$, le diffractogramme obtenu montre que, même si elle apparaît être 
en plus faible proportion, il existe une phase argileuse 2:1 à espace interfoliaire gonflant (fig. 5-a et 9-a). La comparaison des figures $5-c$ et 9 -b indique que le comportement au chauffage de Ech. $<2 \mu \mathrm{m}$ traité par $\mathrm{NaOH} 5 \mathrm{M}$ est semblable à celui de Ech. $<2 \mu \mathrm{m}$ non traité. Les données obtenues à la suite des différents traitements concernent donc l'ensemble des minéraux argileux 2:1.

L'élimination de la kaolinite puis des oxyhydroxydes de fer de Ech. $<0,2 \mu \mathrm{m}$ donne un spectre infrarouge (fig. 4-c) caractéristique des minéraux argileux 2:1. La grande ressemblance, en particulier dans la position et le profil des bandes dues aux groupes $\mathrm{OH}$ de structure, entre le spectre de ces minéraux argileux 2:1 (fig. 4-c) et celui de l'illite du Puy (fig. 4-d) indique qu'il s'agit d'illite. La valeur de la charge est, pour ce type de minéral argileux, de 0,7 à 0,8 par maille et se situe essentiellement en couche tétraédrique (BRINDLEY \& BROWN, 1980). Ce caractère est en accord avec l'analyse faite à partir des résultats de diffraction des RX.

\section{Les oxyhydroxydes métalliques}

Aucun composé du fer n'a été identifié en diffraction des RX même après avoir effectué sur Ech. $<0,2 \mu \mathrm{m}$ le traitement par $\mathrm{NaOH} 5 \mathrm{M}$ qui vise à concentrer les oxyhydroxydes de fer par dissolution sélective des minéraux argileux (KAMPF \& SCHWERTMANN, 1982).

En spectrométrie Mössbauer, les valeurs de l'écart quadrupolaire $2 \varepsilon$ et du déplacement isomérique $\delta$ obtenues à la température ambiante avec Ech. $<2 \mu \mathrm{m}$ indiquent que la totalité du fer présent dans cet échantillon est à l'état $\mathrm{Fe}_{\text {octa }}^{++}$(fig. 10, tabl. 3). En effet, pour $\mathrm{Fe}_{\text {octa. }}^{++}, 2 \varepsilon$ et $\delta$ sont respectivement proches de 0,50 et $0,25 \mathrm{~mm} / \mathrm{s}$ alors que pour $\mathrm{Fe}_{\text {octa }}^{+}$, ces valeurs sont proches ou supérieures à $1 \mathrm{~mm} / \mathrm{s}$ (JANOT et al., 1973 ; JEANROY, 1983). Par ailleurs, l'ajustement d'un sextuplet pour ce spectre obtenu à la température ambiante est apparu non significatif ; l'ensemble du $\mathrm{Fe}_{\text {octa. }}^{++}$possède donc à cette température un comportement superparamagnétique. Ceci indique que si de l'hématite ou de la goethite sont présentes dans Ech. $<2 \mu \mathrm{m}$, elles sont respectivement de taille inférieure à 8 et $15 \mathrm{~nm}$ (JANOT et al., 1973). A $80^{\circ} \mathrm{K}$, l'existence d'un sextuplet est due à la présence d'oxyhydroxydes de fer (fig. 10). La valeur du champ hyperfin indique qu'il s'agit de goethite, elle serait alors de taille com-

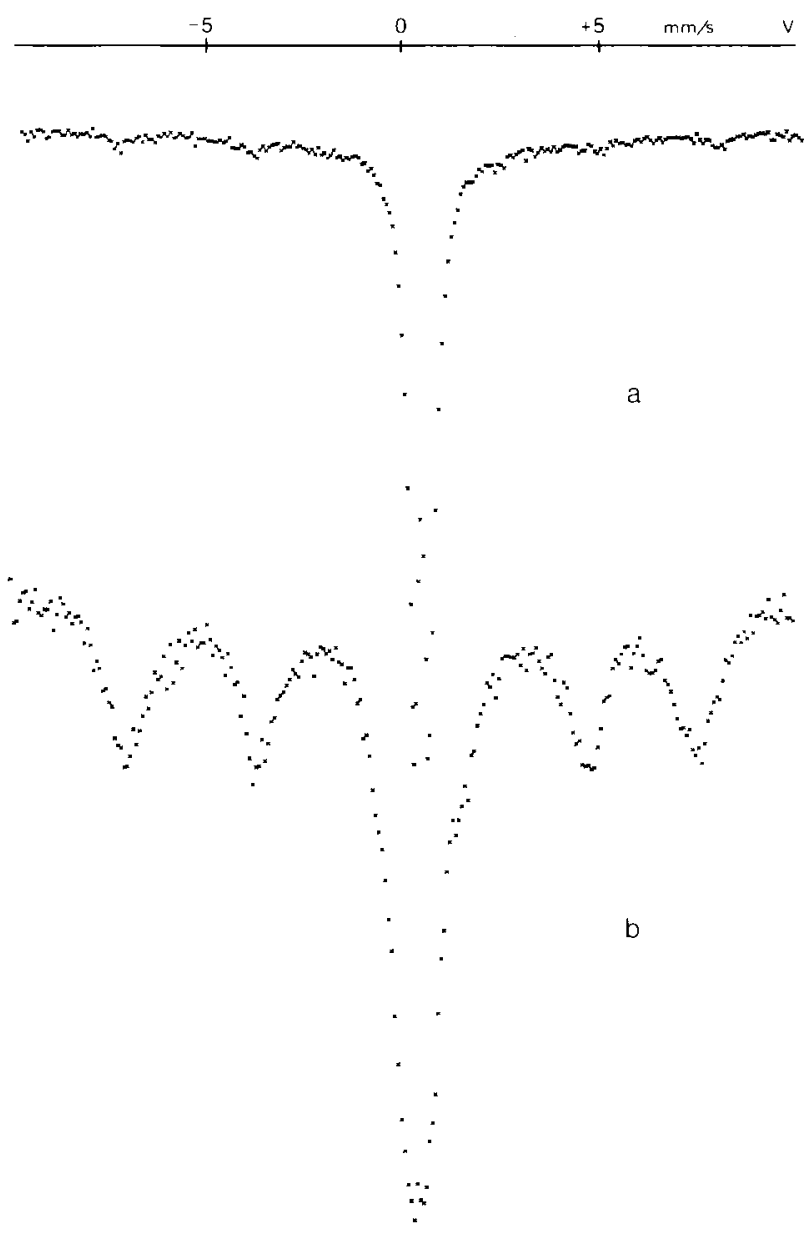

Figure 10

Spectres Mössbauer de Ech. $<2 \mu m:$ (a) température ambiante, (b) $80^{\circ} \mathrm{K}$.

Mössbauer spectra of the $<2 \mu \mathrm{m}$ sample: (a) room temperature, (b) $80^{\circ} \mathrm{K}$.

prise entre 15 et $8 \mathrm{~nm}$ (JANOT et al., 1973). Les ajustements effectués permettent d'estimer qu'elle représente environ 40 p. $100 \mathrm{du}$ fer total. Quant au doublet, représentant les 60 p. 100 restants, il peut s'agir d'hématite ou de goethite respectivement de taille inférieure à 3 et $8 \mathrm{~nm}$, de fer dans les minéraux argileux ou encore de fer appartenant à d'autres composés non identifiables dans de telles conditions expérimentales.

Le spectre de réflexion diffuse de Ech. $<2 \mu \mathrm{m}$ (fig. 7-a) présente une bande d'absorption proche de

TABLEAU 3

Paramètres des spectres Mössbauer obtenus à la température ambiante et à $80^{\circ} \mathrm{K}\left(\delta:\right.$ déplacement isomérique par rapport à la source ${ }^{57} \mathrm{Co}$; 2 : : écart quadrupolaire; $L 1 / 2$ : largeur à demi-hauteur et $H_{i}$ : champ hyperfin).

Mössbauer parameters obtained at room temperature and $80^{\circ} \mathrm{K}\left(\delta:\right.$ isomer shift with respect to $\mathrm{Co}^{57}$ source ; $2 \varepsilon$ : quadrupole splitting $L 1 / 2:$ half way up width and $H_{i}:$ magnetic field).

\begin{tabular}{|c|c|c|c|c|c|c|c|}
\hline & \multicolumn{3}{|c|}{ Doublet } & \multicolumn{4}{|c|}{ Sextuplet } \\
\hline & $\begin{array}{c}\delta \\
\mathrm{mm} / \mathrm{s}\end{array}$ & $\begin{array}{c}2 \epsilon \\
\mathrm{mm} / \mathrm{s}\end{array}$ & $\begin{array}{l}\mathrm{L} \mathrm{l} / 2 \\
\mathrm{~mm} / \mathrm{s}\end{array}$ & $\begin{array}{c}\delta \\
\mathrm{mm} / \mathrm{s}\end{array}$ & $\begin{array}{c}2 \epsilon \\
\mathrm{mm} / \mathrm{s}\end{array}$ & $\begin{array}{l}\mathrm{L} 1 / 2 \\
\mathrm{~mm} / \mathrm{s}\end{array}$ & $\begin{array}{c}\mathrm{H}_{\mathrm{i}} \\
\text { Tesla }\end{array}$ \\
\hline $\begin{array}{l}\text { Température } \\
\text { ambiante }\end{array}$ & 0,26 & 0,59 & 0,46 & - & - & - & - \\
\hline $80^{\circ} \mathrm{K}$ & 0,24 & 0,29 & 0,86 & 0,25 & $-0,14$ & 1,29 & 47 \\
\hline
\end{tabular}


$900 \mathrm{~nm}$ qui est due à une transition électronique du fer $^{++}+$(BOTHOREL, 1983). Cette bande d'absorption est sur le flanc d'un massif d'absorption très intense situé dans le visible. On a, dans une $1^{\text {re }}$ approximation, tracé une droite tangente aux 2 minima et recalculé la position du maximum d'absorption par rapport à cette nouvelle ligne de base (PROST et al., 1983). Nous pouvons aussi, pour mieux tenir compte de la courbure de la ligne de base, extrapoler la courbe et réaliser le même calcul. Les valeurs obtenues à l'issue de ces 2 types de calcul, avant et après traitement de Ech. $<2 \mu \mathrm{m}$ par $\mathrm{NaOH} 5 \mathrm{M}$, sont identiques. La position du maximum d'absorption ainsi déterminée est à $924 \mathrm{~nm}$. On note aussi que la surface de la bande d'absorption est supérieure après traitement à ce qu'elle était avec Ech. $<2 \mu \mathrm{m}$ non traité (fig. 7). Cet accroissement de la surface est en accord avec le fait que le traitement chimique concentre les composés du fer. En raison de la précision limitée du calcul effectué pour déterminer la position du maximum d'absorption, l'apparente absence de déplacement est ici insuffisante pour affirmer que ce traitement concentre les composés du fer sans les modifier. Néanmoins, la position du maximum d'absorption $(924 \mathrm{~nm})$ est en accord avec la présence de goethite dans Ech. < $2 \mu \mathrm{m}$ (HUNT, 1977 ; HUNT \& ASHLEY, 1979 ; BOTHOREL, 1983).

Enfin, la bande d'absorption infrarouge très large centrée sur $3425 \mathrm{~cm}^{-1}$ (fig. 3-a, 4-a) est due à l'eau d'hydratation des argiles (SPOSITO \& PROST, 1982). Celle située à $3160 \mathrm{~cm}^{-1}$ (fig. 3, 4-a et 4-b), qui disparaît lorsque l'échantillon est traité par la méthode de DE ENDREDY (fig. 4-c), est due aux groupes $\mathrm{OH}$ de structure de la goethite (SCHWARZMANN \& SPARR, 1969 ; VERDONCK et al., 1982).

\section{CONCLUSION}

La démarche suivie vise à caractériser les constituants du sol, en particulier ceux qui sont présents en très petites quantités et/ou ceux qui nécessitent, à cause de leur taille ou de leur cristallinité, la mise en œuvre d'autres techniques que celles habituellement utilisées (diffraction des RX, analyses thermiques, ...).

La $1^{\text {re }}$ partie de cet objectif a été atteinte en utilisant des traitements spécifiques (fractionnement granulométrique, dissolutions sélectives) qui permettent l'enrichissement de l'échantillon en l'une ou l'autre des phases existantes. L'application en cascade de ces traitements en augmente l'efficacité. Cette opération est délicate car elle doit être réalisée en évitant de perdre ou d'altérer l'un ou l'autre des constituants que l'on cherche à caractériser. On montre, sur le sol étudié, que la séparation granulométrique des particules $<0,2 \mu \mathrm{m}$ permet l'élimination des grains de quartz qu'il contient.

Les échantillons obtenus à partir de ces prétraitements ont été examinés à l'aide de différentes techniques. L'exemple étudié a permis de montrer comment l'utilisation des spectrométries infrarouge et Mössbauer permet de préciser la nature de certains constituants qu'il n'est pas possible de caractériser convenablement par diffraction des RX. C'est ainsi que l'illite et la goethite ont pu être identifiées dans l'échantillon étudié.

Reçu le 11 juillet 1985 Accepté le 17 avril 1986.

\section{REMERCIEMENTS}

Les auteurs remercient Mme TRONC (E.N.S.C.P. Paris) pour la réalisation et l'interprétation des spectres Mössbauer. Un grand nombre de personnes de la Station de Science du Sol de l'I.N.R.A. Versailles ont contribué à la réalisation de ce travail ; qu'elles soient remerciées ici pour leur aide.

\section{RÉFÉRENCES BIBLIOGRAPHIQUES}

Baize D., 1971. Contribution à l'étude des sols des plateaux jurassiques de Bourgogne. Thèse $3^{\mathrm{e}}$ cycle, Univ. Paris, $156 \mathrm{p}$.

Baize D., 1972a. Les sols développés dans la couverture des plateaux jurassiques de Bourgogne. Pédogenèse et origine. Bull. Assoc. fr. Etude Sol, 1, 37-43.

Baize D., 1972b. Micromorphologie des «terres d'Aubues » des plateaux de Bourgogne. Bull. Assoc. fr. Etude Sol, 1-2, 63-71.

Baize D., 1976. Notice explicative de la carte pédologique de France à $1 / 100000$. Feuille de Tonnerre. I.N.R.A., Paris, 243 p.

Bothorel A., 1983. Télédétection des ressources terrestres : signatures spectrales (visible, proche infrarouge) de roches et de minerais en zone aride; application à trois types de minéralisations au Maroc. Thèse $3^{e}$ cycle, Univ. Paris VI, 145 p.

Brindley G. W., Brown G., 1980. Crystal structures of clay minerals and their X-ray identification. Mineral Soc. G. B., Monograph. 5, London, $495 \mathrm{p}$.

Bruand A., 1985. Contribution à l'étude de la dynamique de l'organisation de matériaux gonflants. Application à un matériau provenant d'un sol argilo-limoneux de l'Auxerrois. Thèse $3^{\text {e }}$ cyclẹ, Univ. Paris VII, $226 \mathrm{p}$
Caillère S., Henin S., Rautureau M., 1982. Minéralogie des argiles. Masson, Paris, Tomes 1 et 2, 184 et 189 p.

Deb B. C., 1950. The estimation of free iron oxides in soils and clays and their removal. J. Soil Sci., 1 (2), 212-220.

Decarreau A., 1983. Etude expérimentale de la cristallogenèse des smectites. Sci. géol. Mem., 74, 184 p.

De Endredy A., 1963. Estimation of free iron oxides in soils and clay by a photolytic method. Clay Miner. Bull., 29 (5), 209-217.

Farmer V. C., 1974. The infrared spectra of minerals. Mineral. Soc., Monograph 4, London, 539 p.

Hunt G. R., 1977. Spectral signature of particulate minerals in the visible and near-infrared. Geophysics, 42 (3), 501-513.

Hunt G. R., Ashley R. P., 1979. Spectra of altered rocks in the visible and near-infrared. Econ. Geol., 74, 1613-1629.

Hunt G. R., Salisbury J. W., Lenhoff C. J., 1973. Visible and near infrared spectra of minerals and rocks : VI - Additional silicates. Mod. Geol., 4, 85-106.

Janot Ch., Gibert H., Tobias Ch., 1973. Caractérisation de kaolinites ferrifères par spectrométrie Mössbauer. Bull. Soc. fr. Mineral. Cristallogr., 96, 281-291. 
Jeanroy E., 1983. Diagnostic des formes du fer dans les pédogenèses tempérées. Evaluation par les réactifs chimiques d'extraction et apports de la spectrométrie Mössbauer. Thèse Univ. Nancy I, $169 \mathrm{p}$.

Kampf N., Schwertmann U., 1982. The 5-M-NaOH concentration treatment of iron oxides in soils. Clay Miner., 30 (6), 401-408.

Pedro G., 1967. Commentaires sur la classification et la nomenclature des minéraux argileux (A propos de la deuxième conférence internationale sur les argiles - Jérusalem, 1966). Bull. Groupe $f r$. Argiles, 19, 69-86.

Prost R., King C., Lefebvre d'Hellencourt T., 1983. Propriétés de réflexion diffuse des pâtes de kaolinite en fonction de leur teneur en eau. Clay Miner., 18, 193-204.

Rautureau M., Tchoubar C., 1974. Précisions concernant l'analyse structurale de la sépiolite par microdiffraction électronique. $C . R$. Acad. Sci., Paris, Sér. B, 278, 25-28.
Robert M., 1975. Principes de détermination qualitative des minéraux argileux à l'aide des rayons $\mathrm{X}$. Problèmes particuliers posés par les minéraux argileux les plus fréquents dans les sols des régions tempérées. Ann. agron., 26 (4), 363-399.

Rouxhet P. G., Ngo Samudacheata, Jacobs H., Anton O., 1977. Attribution of the $\mathrm{OH}$ stretching bands of kaolinite. Clay Miner., 12, $171-179$

Schwarzmann E., Sparr H., 1969. Die Wasserstoffbrückenbindung in Hydroxiden mit Diasporstruktur. Z. Naturforsch., 246, 8-11.

Schwertmann U., 1964. The differentiation of iron oxides in soil by extraction with ammonium oxalate solution. Z. Pflanzenernaehr. Dueng., Bodenkd., 105, 194-202.

Sposito G., Prost R., 1982. Structure of water adsorbed on smectites. Chem. Rev., 82 (6), 553-573.

Verdonck L., Hoste S., Roelandt F. F., Van der Kelen G. P., 1982. Normal coordinate analysis of $x-\mathrm{FeOOH}$. A molecular approach. J. mol. Struct., 79, 273-279. 